\title{
Complex Mapping of 3DCG Models with Julia Set and Mandelbrot Set
}

\author{
Ningping Sun and Fuko Nakagami
}

\begin{abstract}
We introduced color interpolation according to the diverging speed into the flat images of Julia set and Mandelbrot set for making shading-reflecting like effect. We named our method "Complex Mapping". In this paper we shall describe our color interpolation algorithm and the method how to map Julia set and Mandelbrot set on the inside and outside of the surface of 3DCG models.
\end{abstract}

Keywords-Julia set, Mandelbrot set, fractal, complex mapping, 3DCG

\section{Introduction}

Julia set and Mandelbrot set are very famous and elaborate fractals that are self-similar with the iteration of a function of complex variable. These two sets provide a most striking illustration of how an apparently simple process can lead to highly intricate set. Different to the existing results we introduced color interpolation according to the diverging speed into the flat images of Julia set and Mandelbrot set for making shading-reflecting like effect. We named our method "Complex Mapping" ([1]). Using the complex mapping we have developed some new approaches to render the inside and outside of the surface of primitive models such as Riemann sphere, cylinder, cube, cone and torus, in which the ordinary curved surface modeling and texture mapping are not needed any more.

In this paper we first discuss the properties of Julia set and Mandelbrot set based on some results of our experiments. In the second part, we describe our color interpolation algorithm. Finally we explain some ways how to map the interpolated Julia set and Mandelbrot set on the inside and outside of the surface of models. Some result of verification experiments will be provided also.

Ningping Sun

Kumamoto College, National Institute of Technology Japan

\section{Interpolating Gradation Algorithm of Two Fractals}

We first discuss the properties of Julia set and Mandelbrot set based on some results of our experiments and new ideas.

\section{A. Julia Set and Mandelbrot Set}

Julia set was investigated by the French mathematicians Pierre Fatou and Gaston Julia at the beginning of the 20th century. Mandelbrot set was defined and named due to Adrien Douady, in tribute to the mathematician Benoit Mandelbrot in 80's. Julia set and Mandelbrot set are the set of complex numbers. In the complex plane a point, $z$, can be represented as $z=x+y i$, where $x$ is a real number and $y$ is an imaginary number. We can get these two sets from (1). These two sets are also called fractals because of their selfsimilarity, so that we can define them as (2) based on their recursive features as well.

$$
\begin{aligned}
& \text { Quadratic function: } \quad f_{c}(z)=z^{2}+c \\
& \text { Recursive function: } \quad z_{n}=z_{n-1}^{2}+c \\
& \text { Julia set: } z_{0}=x+y i, c=a+b i, a, b \text { : constant } \\
& \text { Mandelbrot set: } z_{0}=0, c=x+y i
\end{aligned}
$$

Julia set and Mandelbrot set are applied in mathematical visualization widely as the best-known fractals. Actually, we iterate the calculation of (2) from negative 2 to 2 for $x$ and $y$ in the complex plane. For Julia set we calculate from a starting point $z o$ and use two constants $a$ and $b$; a little differently, we calculate Mandelbrot set from $z_{0}=0$ and the value of $c$ is as same as the starting point. The number of iterations, $n$, is very important in this recursive calculation that will be described in details below. We give two examples in Figure 1 and 2. In the Julia set of Figure 1, the region is from $-0.599+0.664 i$ to $0.712-0.648 i$, the constant is $c=-1.06+0.25 i$, and $n=512$. In the Mandelbrot set of Figure 2, $c=-2.0+0.463 i$, and $n=$ 512

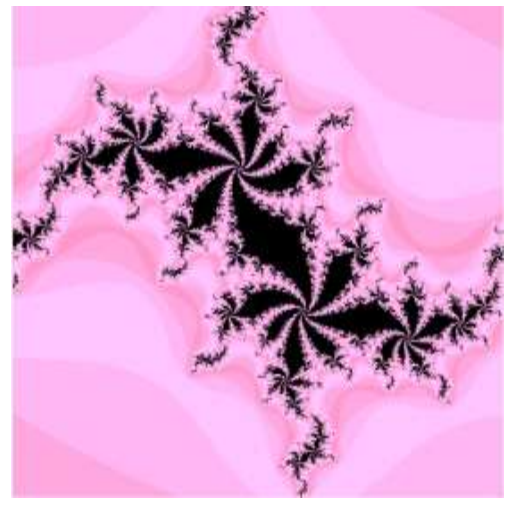

Figure 1. Julia set $(c=-1.06+0.25 i)$
Fuko Nakagami

Kumamoto College, National Institute of Technology Japan 
Proc. of Sixth International Conference On Advances in Computing, Electronics and Electrical Technology - CEET 2016

Copyright (C) Institute of Research Engineers and Doctors. All rights reserved.

ISBN no. 978-1-63248-109-2 doi: 10.15224/ 978-1-63248-109-2-25

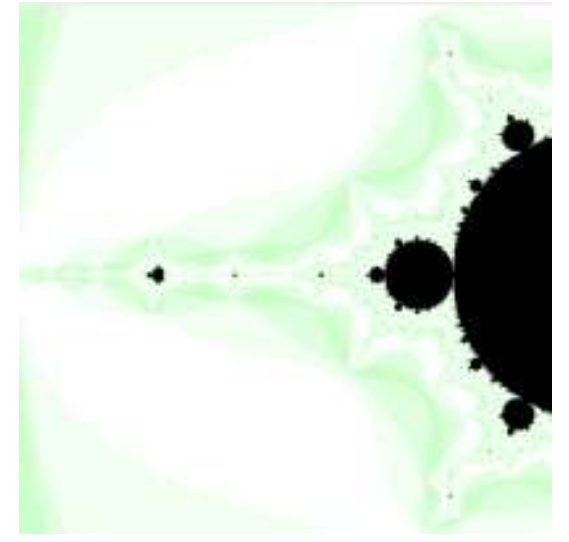

Figure 2. Mandelbrot set $(c=-2.0+0.463 i)$

\section{B. The relationship between /z/ and $n$}

When we calculate the two fractals the point that is very close to the origin $(0,0)$ what it tends to do is spiral inward as we iterate (2). Its value of $|z|$ is becoming smaller and smaller and never goes outside of 2 , we call this point convergence point and color it black. Actually, Julia set and Mandelbrot set are sets of the black points. If the value of $|z|$ of a point is becoming larger and larger when we apply (2), and it eventually goes outside of 2 , the point is called divergence point vice versa. When $|z|>w, w$ is a designated diverging threshold value, we stop performing (2) and color the point according to its number of iterations what it just took.

We are interested in those divergence points of Julia set and Mandelbrot set for their potential possibilities. How to use them for 3DCG rendering? We want to know how is the shape of the two fractals determined by the number of iterations. We have investigated and carried out experiments to clarify the relationship between $|z|$ and $n$. For convenient, we set $w=4$ as divergence condition in our experiments. Some results are shown in TABLE I and Figure 3 and 4.

TABLE I. THE MAXIMUM AND MINIMUM VALUES OF $|\mathrm{z}|$

\begin{tabular}{|c|l|r|r|r|r|}
\hline number $\begin{array}{c}\text { nof } \\
\text { of } \\
\text { iterations }\end{array}$ & \multirow{2}{*}{ color } & \multicolumn{2}{|c|}{ Mandelbrot set } & \multicolumn{2}{c|}{ Julia set } \\
\cline { 2 - 6 } & & $|Z|$ min & $|Z|$ max & $|Z|$ min & $|Z|$ max \\
\hline 1 & gray & 4 & 8 & 4.00001296 & 69.03128341 \\
\hline 2 & red & 4 & 35.93339245 & 4.00012507 & 26.8681285 \\
\hline 3 & green & 4.000081332 & 33.1441278 & 4.000193819 & 26.79776728 \\
\hline 4 & blue & 4.002401201 & 34.36061306 & 4.000481271 & 26.78019115 \\
\hline 5 & yellow & 4.002558867 & 33.72790265 & 4.000549548 & 26.6971431 \\
\hline
\end{tabular}

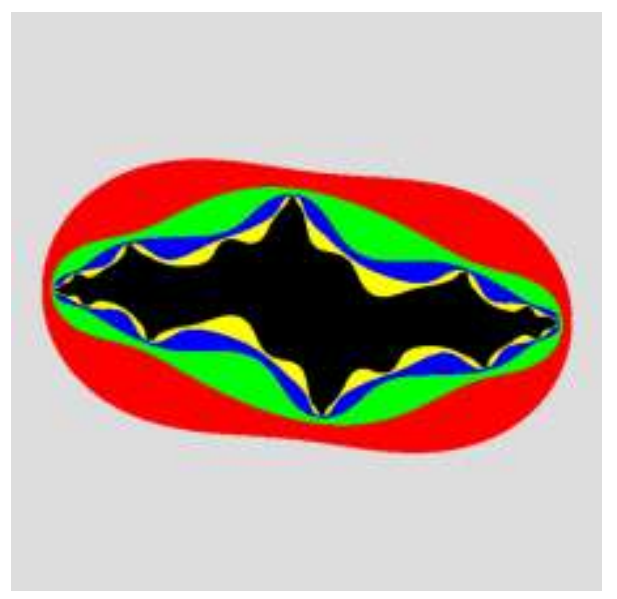

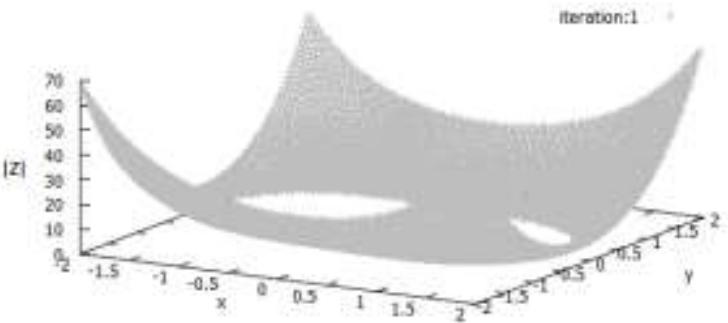
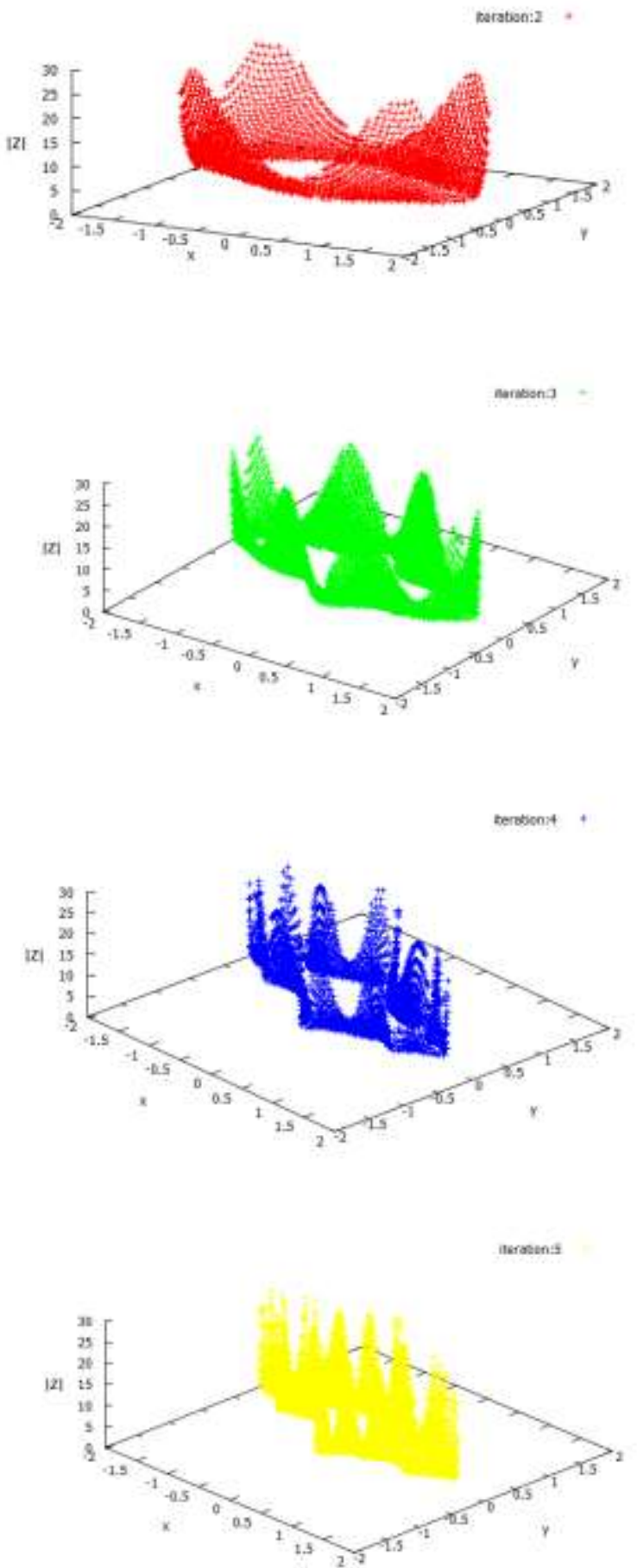

Figure 3. Values of $|z|$ of Julia set $(c=-1.16+0.25 i)$ at different $n$ 
Proc. of Sixth International Conference On Advances in Computing, Electronics and Electrical Technology - CEET 2016

Copyright (C) Institute of Research Engineers and Doctors. All rights reserved. ISBN no. 978-1-63248-109-2 doi: 10.15224/ 978-1-63248-109-2-25
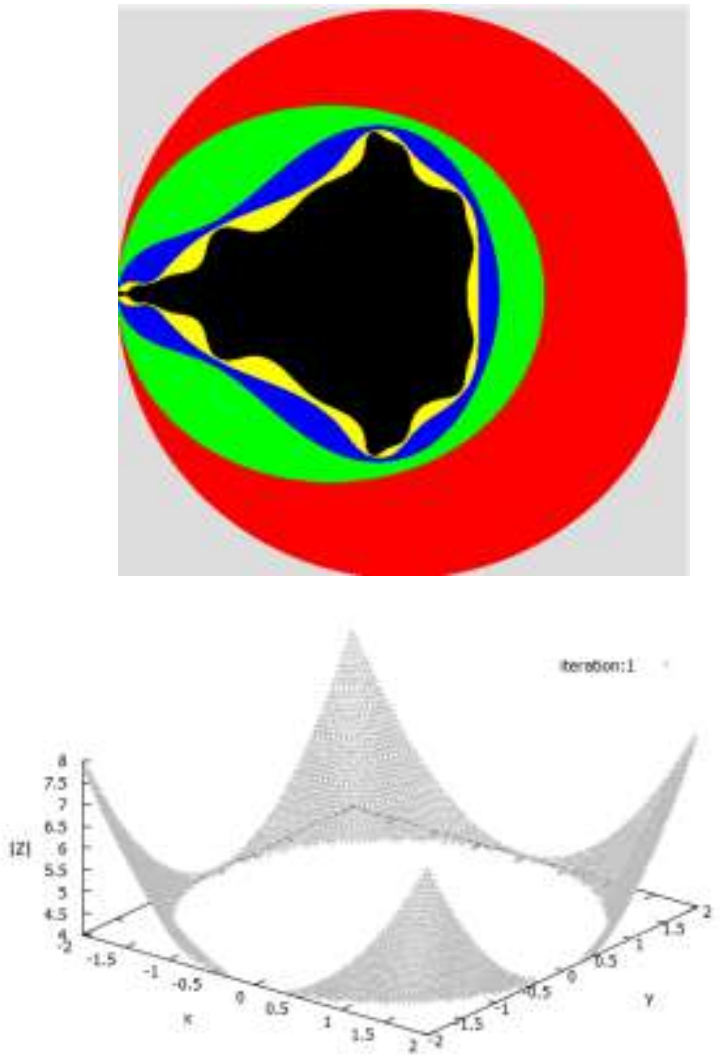

earation:2 -
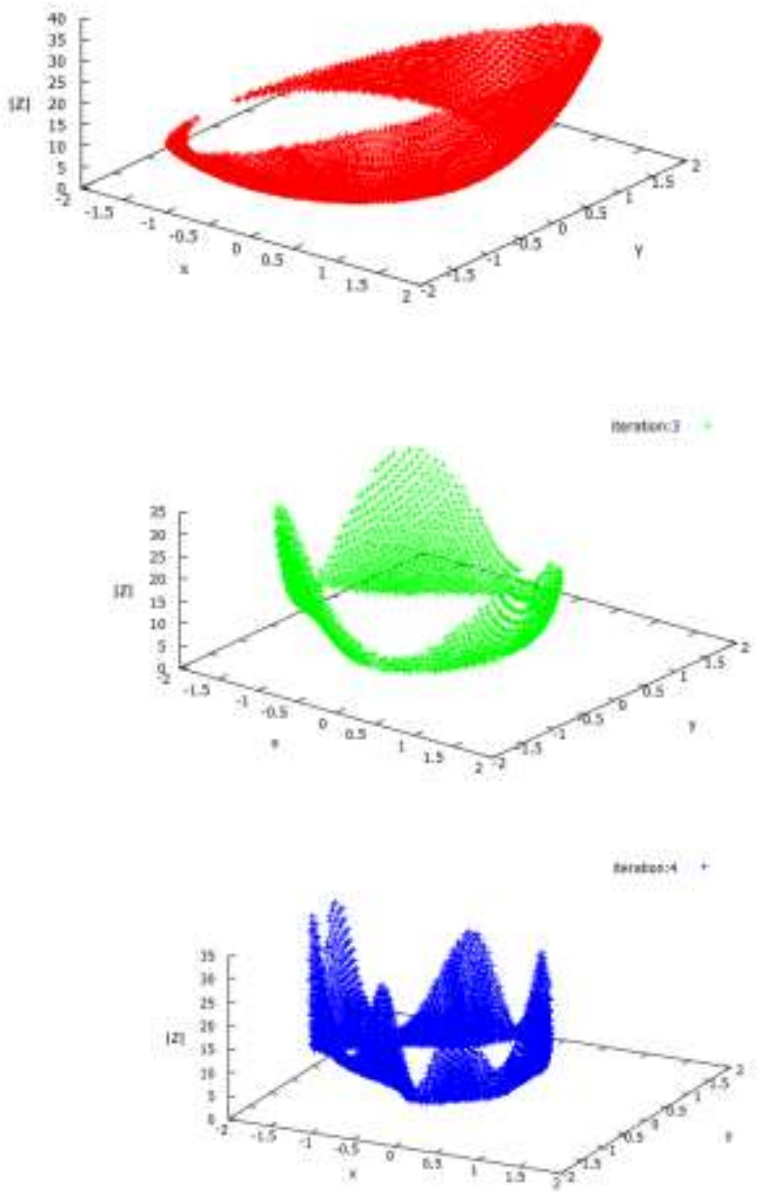

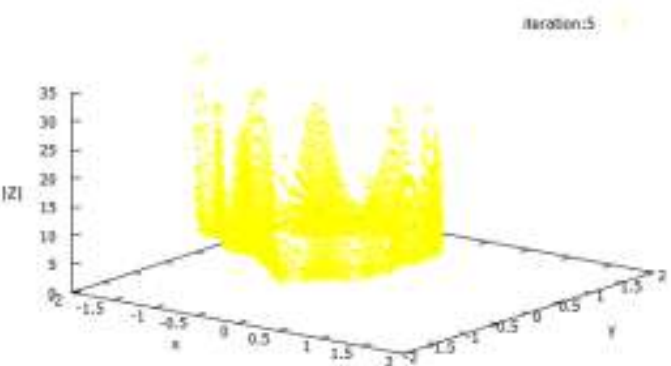

Figure 4. Values of $|\mathrm{z}|$ of Mandelbrot set $(c=-2.0+2.0 i)$ at different $n$

We iterate the calculation of (2) from negative 2 to 2 for $x$ and $y$ on the complex plane. As shown in TABLE I we color the point gray if its value of $|z|$ becomes greater than $w$ by performing (2) once, similarly red for twice, and so on. From our experiments we found that the points having same $n$ always crowd together in a same region bounded with the related $|z n| \min$ and $|z n| \max$. Meanwhile, for a given $n$ each point has its $|z n|$ itself differently depends on its coordinates and $c$ around an elliptical oval.

The same phenomenon also happens in the case of Figure 5, where a Mandelbrot set is in the region from $1.936+0.183 i$ to $-1.576-0.176 i$ and $\mathrm{c}=-1.936+0.183 i$. Here the number of iterations is from 1 to 256 . In the complicated graphic figure every $n$ keeps its territory accurately and never confound with its neighbors each other, which is the same as other results of our experiments.
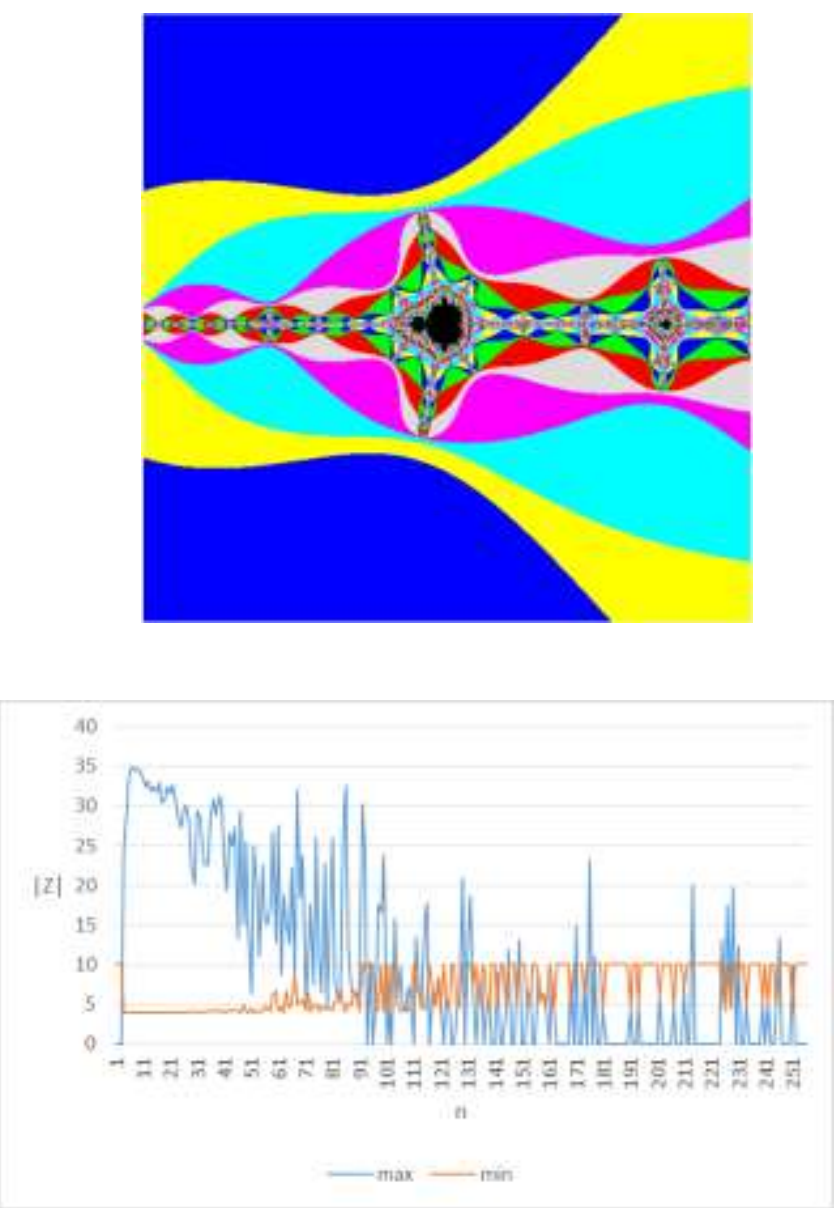

Figure 5. The relationship between $|z|$ and $n$ 
Proc. of Sixth International Conference On Advances in Computing, Electronics and Electrical Technology - CEET 2016

Copyright (C) Institute of Research Engineers and Doctors. All rights reserved. ISBN no. 978-1-63248-109-2 doi: 10.15224/ 978-1-63248-109-2-25

\section{Interpolating Gradation Algorithm}

We proposed a color interpolation method to interpolating gradation in each $n$th territory based on our experiment results. For each $n$ we first find out $|z n|$ min and $|z n|$ max over the whole $n$th region. Continuously, for each point we calculate the following two coefficients: convex coefficient and concave coefficient by (3).

$$
\begin{array}{ll}
\text { Convex coefficient: } & g_{n}=\left|z_{n}\right| /\left|z_{n}\right|_{\max } \\
\text { Concave coefficient: } & b_{n}=\left|z_{n}\right|_{\min } /\left|z_{n}\right|
\end{array}
$$

Multiplying the convex coefficients $g_{n}$ and the color of pixel within the $n$th area, and doing this over all of image of Julia set or Mandelbrot set, we will obtain somewhat like shading and reflecting effect as shown in the left side of Figure 8 . Because $b_{n}$ is a concavity parameter for the points of $n$th region we can use it to represent the back of surface as shown in the right side of Figure 8.

We are interested in mapping the color interpolated images onto the outside and inside of surfaces of models instead of general texture mapping.

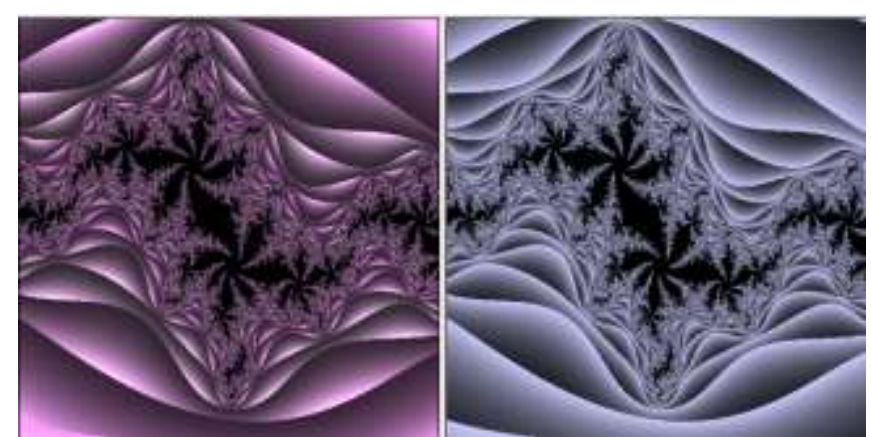

Figure 6. Interpolated gradation of the Julia set of Figure 1

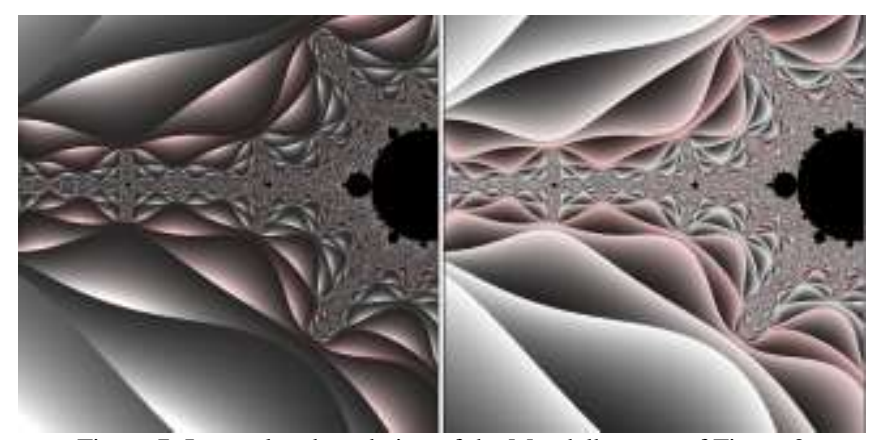

Figure 7. Interpolated gradation of the Mandelbrot set of Figure 2

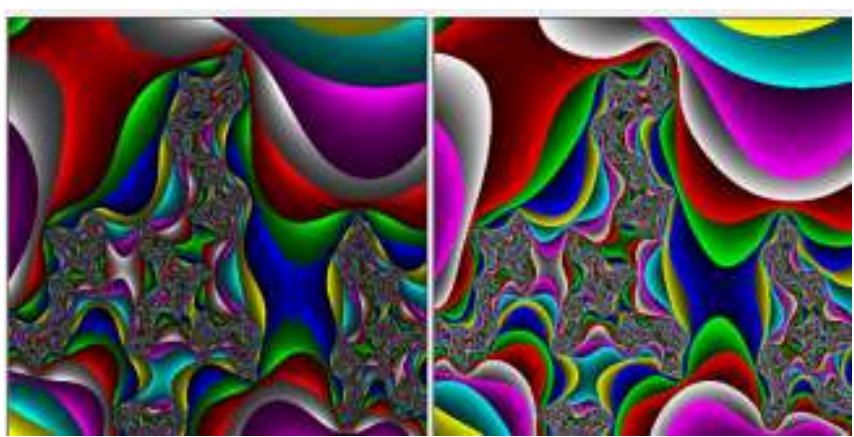

Figure 8. Interpolated gradation of Julia set $(c=-1.08+0.35 i)$

\section{Complex Mapping with Two Interpolated Fractals}

The color of a point is multiplied by convex parameter when we render outside of surface of a $3 \mathrm{D}$ model. In the case of rendering the back of surface the color is multiplied by the concave parameter. Different from the general texture mapping, "complex mapping" not only renders the outside of model surface but also renders its inside using the same interpolated fractals and ether same or different colors. At the same time, the converge portions consisted of the black points in Julia set and Mandelbrot set are not to be rendered, so that the inside of concavity can be seen through these artificial cavities.

Using the complex mapping we have developed some new approaches to render the inside and outside of surface of primitive models such as Riemann sphere, cylinder, cube, cone, and torus. The ordinary curved surface modeling and texture mapping are not needed any more in our mapping.

\section{A. Riemann sphere}

Riemann sphere is one of the techniques to extend the complex plane by adding one infinity point on the plane. Coordinates on the sphere, $(X, Y, Z)$ can be calculated by (4) where $a$ is the radius of the sphere, $z=x+y i$ is a point on the complex plane.

$\mathrm{X}=\frac{a^{2}(z+\bar{z})}{a^{2}+|z|^{2}}, \quad \mathrm{Y}=\frac{a^{2}(z-\bar{z})}{i\left(a^{2}+|z|^{2}\right)}, \quad \mathrm{Z}=\frac{a|z|^{2}-a^{3}}{a^{2}+|z|^{2}}$

Some results of mapping on Riemann sphere are shown in Figure 9, 10, and 11.
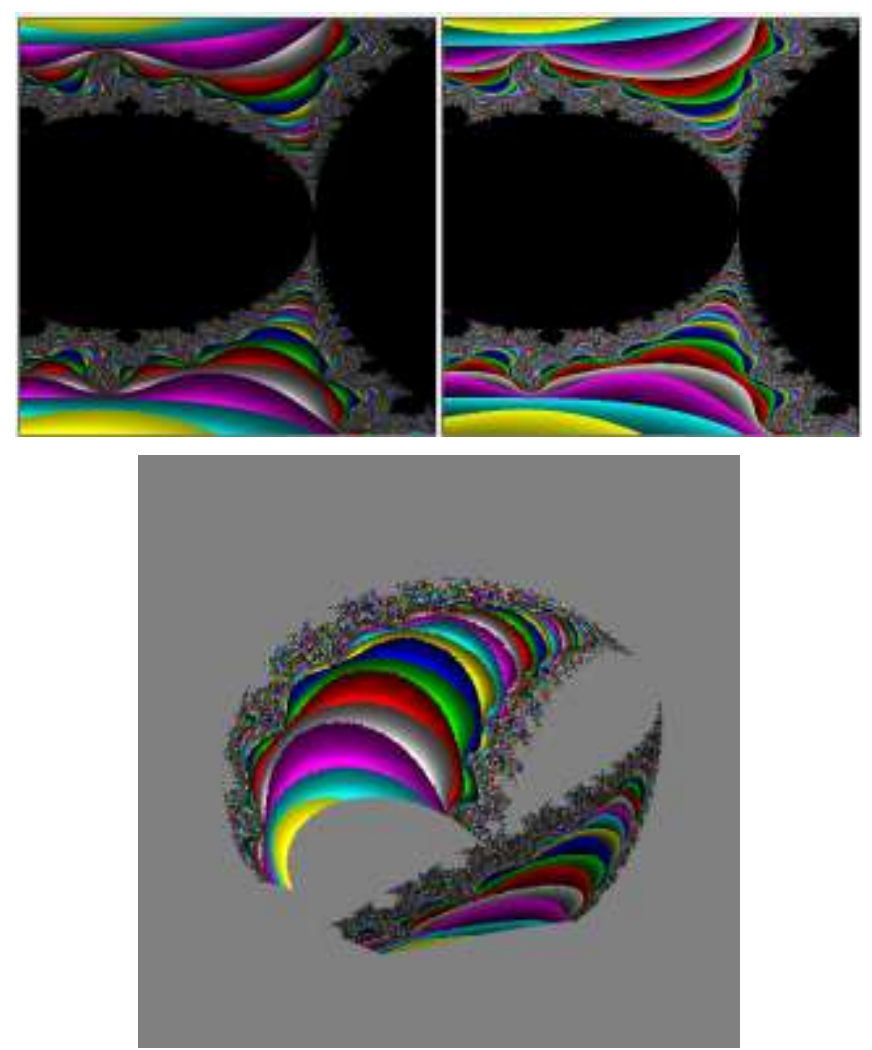

Figure 9. Complex mapping on Riemann sphere with interpolated Mandelbrot set 
Proc. of Sixth International Conference On Advances in Computing, Electronics and Electrical Technology - CEET 2016

Copyright (C) Institute of Research Engineers and Doctors. All rights reserved. ISBN no. 978-1-63248-109-2 doi: 10.15224/ 978-1-63248-109-2-25

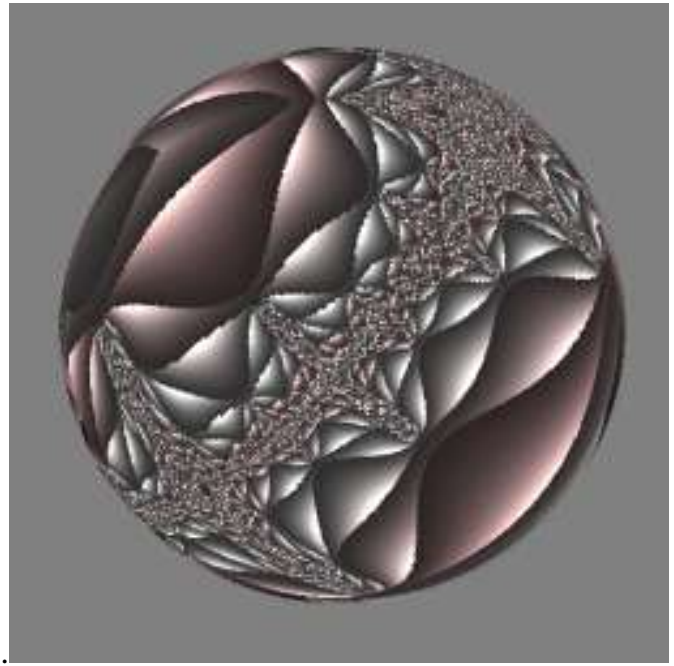

Figure 10. Riemann sphere mapped with Mandelbrot set of Figure 7

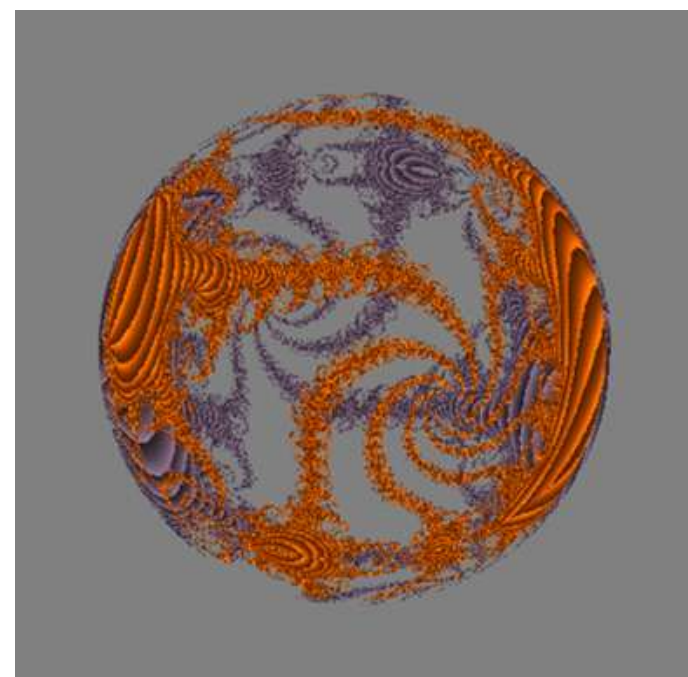

Figure 11. Complex mapping on Riemann sphere with Julia set

\section{B. Torus}

Using (5) we can render a torus of Fig 12. In (5) $R=0.5$, $r=0.3,0 \leq \varphi \leq 2 \pi, 0 \leq t \leq 2 \pi$.

$$
\begin{aligned}
& \mathrm{X}=\mathrm{R} \cos t+r \cos \varphi \cos t \\
& \mathrm{Y}=\mathrm{R} \sin t+r \cos \varphi \sin t \\
& \mathrm{Z}=\mathrm{r} \sin \varphi
\end{aligned}
$$

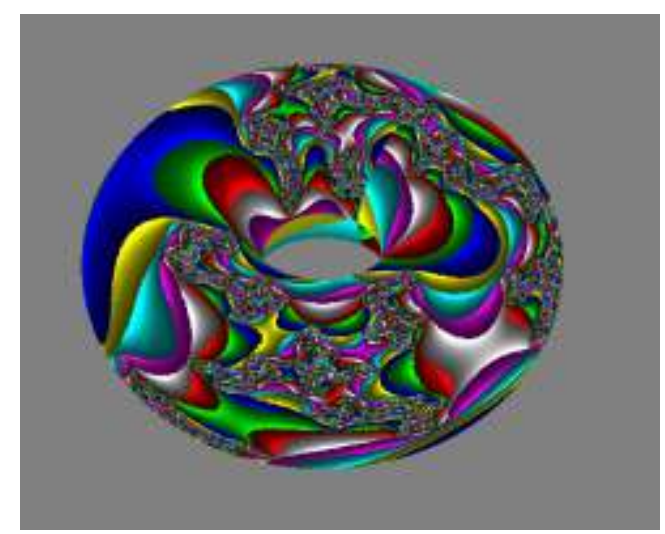

Figure 12. Torus mapped with Mandelbrot set of Figure 8
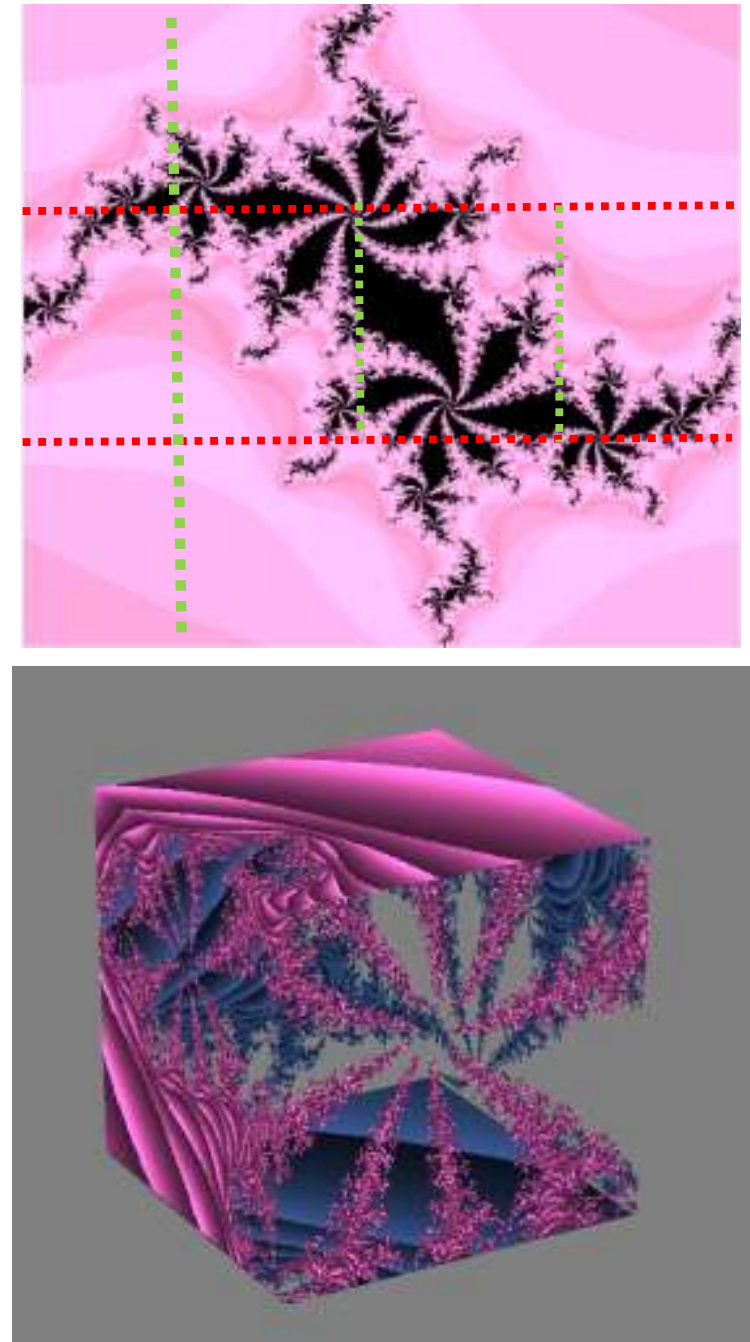

Figure 13.Complex mapping on tube with Julia set of Figure 6

\section{Cube}

We divide the interpolated image as doing in the top of Figure 13 by the dotted lines, then map the interpolated point of fractal onto the corresponding surface of cube by (6), where edge is the length of an edge. A cube as Figure 13 can be rendered.

$$
\begin{aligned}
& \mathrm{X}=\frac{\text { edge }}{\sqrt{2}} \cdot \cos \frac{\pi}{4} \\
& \mathrm{Y}=\frac{\text { edge }}{\sqrt{2}} * \sin \frac{\pi}{4}
\end{aligned}
$$

\section{Iv. Conclusion}

The proposed interpolating gradation algorithm of Julia set and Mandelbrot set works for rendering these two fractals on the outside and inside of surface of 3DCG models. Complex mapping with Julia set and Mandelbrot set onto primitive models gave us significant hints to expand and apply the method to models having arbitrary shapes.

\section{References}

[1] Ningping Sun, Ryo Miyazaki, Naoki Yoshida, Complex Mapping with the Interpolated Julia Set and Mandelbrot Set, Technical Sketches and Poster, 2010, SIGGRAPH Asia 2010, ISBN 978-14503-0439-9/10/0012. 